\title{
STRUM: A SEMANTIC TRUST-BASED MINER MODEL FOR DETECTING INFLUENTIAL SPREADERS IN SOCIAL NETWORKS
}

\author{
Heba M. Wagih ${ }^{1}$, Hoda M. O. Mokhtar ${ }^{2}$ and Samy S. Ghoniemy ${ }^{1}$ \\ ${ }^{1}$ The British University in Egypt, Cairo, Egypt \\ ${ }^{2}$ Cairo University, Cairo, Egypt
}

\begin{abstract}
Social Networks have become embedded in every aspect of today people's life. The success of online social networks has a significant role in shaping trust and influence among participants. They are continuing to gain ground every day, opening the opportunity for several research movements. Great attention is now given for identifying influential spreaders in many application domains as recommendation systems, viral marketing, epidemic control and others. Most of the recent research focused only on the network structure with its geospatial information disregarding the semantics underlying these networks. In this paper, we propose a semantic trust-based miner model (STRUM) that efficiently detects influential spreaders in social networks for enhancing recommendation services. STRUM considers both geospatial information and semantic information in defining each user in the social network. Different experiments have been conducted to verify and validate the proposed algorithm against the latest published using two real location based social networks namely; Brightkite and Weeplaces are used in the experiments.
\end{abstract}

\section{KEYWORDS}

Social Networks, Recommendation Systems, Influential Spreaders

\section{INTRODUCTION}

The proliferation of social network applications is significantly observable in today people's life. In social networks as Facebook, Twitter and others, people have large number of related connections with a varying number, type and strength from one person to another. The use of such applications leads to the generation of a colossal sum of data that opens new research opportunities and challenges to serve people and communities in many different domains. Among which is the epidemic control, recommendation systems, viral marketing and others.

Recommender systems have proven to be an important application that utilizes the current information diffusion in providing users with more customized information services. Location-based recommender applications are newly introduced applications where people can share information about their physical location as well as their common interests and activities. Rodríguez Hernández et al. (2005) presented an important survey that summarizes various location recommendation systems that were developed in different domains as tourism, news and shopping.

An important aspect that directs the recommendation process is trust. People are usually influenced by their trustworthy friends and are more likely to accept recommendations from their trusted friends rather than from random sources. Trust in social networks is a new topic with lots of unanswered questions. Jaouadi and Romdhane (2016) studied two types of social trusts namely; conversational and propagation trust. They develop statistical measures based on the timing and sequence of exchanged messages between users to quantify these trust types. Trust-aware recommender systems have recently gained a special attention in developing recommendation applications. Employing trust information has proven a noticeable enhancement in recommender systems where both the accuracy of recommendation as well as the users' experience are greatly improved. 
Tremendous effort has been recently done to answer many research challenges that have been raised to understand and analyze the dynamics of social networks such influential spreaders which is part of our proposed research. Since detecting influential nodes has never been a minor errand and has an important role in the recommendation process. Thus, in recent years, many influential identification algorithms were introduced such as Degree Centrality (DCM), Betweenness Centrality (BCM), Closeness Centrality (CCM) as cited in Landherr et al. (2010), K-core decomposition as stated in Alvarez-Hamelin et al. (2005), and others. Bonchi (2011) presented an exhaustive survey on different algorithms introduced for detecting influential nodes and how information is propagated in social networks. Zhao et al. (2017) introduced another approach for influential detection known as the local centrality with a coefficient (CLC) algorithm.

Although this research problem has made noticeable strides in past few years, however most of the literature has been focusing only on the social network structure disregarding the importance of semantics aspects that describe these social networks.

Motivated by the strong impact of this research direction in different application domains, we follow the attempts introduced in previous works and present the following contributions:

1. Introduce a Semantic Trust-Based Miner Model (STRUM) for enhancing location recommendation services as well as detecting influential nodes. STRUM is an enhanced version of the proposed algorithm in Wagih et al. (2019) where both the network structure and the relevant semantic information associated are considered.

2. Investigate the role of influential nodes in the recommendation process and specifically in location recommendation.

3. Experimentally evaluate the proposed STRUM with other conventional algorithms on real social network dataset.

The rest of this paper is structured as follows: section 2 presents some related works to both the structural and semantical approaches for influential nodes detection, recommender systems and trusted social networks. In section 3, the semantic trust-based miner model is proposed. In section 4, we provide an experimental evaluation of the proposed algorithm. Finally, section 5 concludes and proposes directions for future work.

\section{RELATED WORK}

In recent years, several types of recommender systems have been proposed, among which are the location-based recommendation systems. Such systems deal with personalized and automatic data retrieved from different sources of information. Detecting trusted influential spreaders in these systems plays an important role in enhancing the quality of the provided recommendation services. Recently many researches have been focusing on discovering vital node as presented by Wei et al. (2018), Wang et al. (2017) and Kim et al. (2014).

Song et al. (2017) proposed a recommendation model named TrustTR based on trust relations and item ratings. The model counts trusted friends' recommendations, item reputation and user history ratings in providing recommendations. The authors experimentally proved that their model provides higher accuracy compared to other conventional recommendation models. Yang et al. (2017) proposed a TrustMF model which integrates both a truster model with a trustee model to address the cold start problem. They proved experimentally that their model outperforms other trust-based models. Recently Davoudi and Chatterjee (2018) introduced a new algorithm for modeling an accurate recommender system. The proposed algorithm considered both finding the relevant items for each user based on his/her interests as well as the social interactions between users. The authors used the probabilistic matrix factorization method to predict user rating for products and rating-based and connection-based similarity measurements for modeling similarity.

Few new researches started to consider the effect of utilizing the semantic information associated with each user in community detection as in Ben Amor (2016) as well as in discovering influential nodes as in Wagih et al. (2019), Hafiene and Karoui (2017), and Jaouadi and Romdhane (2016). Wagih et al. (2019) proposed a semantic based algorithm for detecting influential diffusion in social networks. The proposed algorithm is an extension to the work introduced by Wagih et al. (2019). The authors compared their algorithm with other prior models and the experiments showed a noticeable enhancement in detecting the influential nodes in the network. Jaouadi and Romdhane (2016) proposed a new algorithm called DIN which combines both the structure and the semantic aspects of the network to detect influential nodes. The authors validated their algorithm using artificial networks as datasets however the performance of their algorithm has not been experimented on real social networks. 


\section{SEMANTIC TRUST-BASED MINER MODEL}

In this section, we propose a new model known as Semantic Trust-Based Miner Model (STRUM) for enhancing location recommendation services as well as discovering influential spreaders in social network. The proposed STRUM model is an extended version to the work proposed by Wagih et al. (2019). STRUM proposes solutions to some of the problems that were not addressed in Wagih et al. (2019) as creating a trusted social network and eliminating old visited locations that may affect the recommendation process. STRUM consists of three main Modules; trust calculation module, location mining module and semantic-based influence calculation module. Figure 1 shows the proposed framework. In the following subsections, we introduce each of these modules in details.

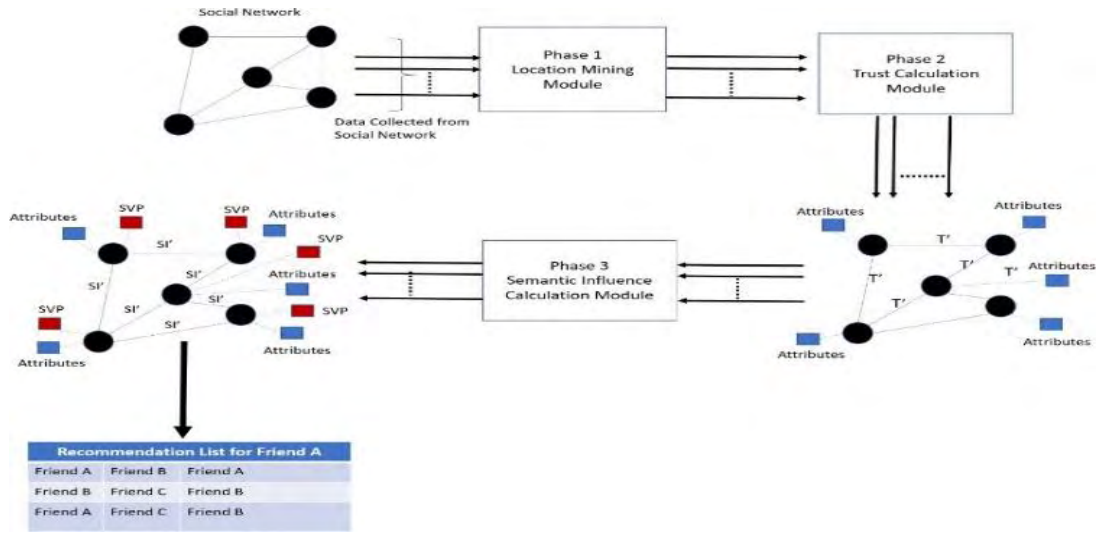

Figure 1. Proposed framework for semantic trust-based miner model

\subsection{Location Mining Module}

In this module, we extract all the visited locations for each user in the social network. For each visited location, we retrieve the visited frequency by each user and the check-in time. User's preferences and choices might change over time; therefore, it is important to eliminate all places that were checked-in before a certain time threshold as such places could falsely affect the recommendation process. Since the proposed model aims at enhancing location recommendation services, thus location filtering module is crucial to create a more trusted network. Figure 2 shows an example of visited locations log table after filtration from Brightkite dataset.

$$
\operatorname{Imp}(\operatorname{Loc})=\left\{\begin{array}{l}
1, \text { VisitedTime }<\text { Threshold } \\
0, \text { VisitedTime } \geq \text { Threshold }
\end{array}\right.
$$

\begin{tabular}{|c|c|c|c|c|c|c|c|}
\hline Location & User & Check-in Time & Frequency & & & & \\
\hline Hotel & 1 & 2009-05-15T14:29:15Z & 1 & Location & User & Check-in Time & Frequency \\
\hline School & 1 & 2009-05-15T11:42:53Z & 2 & Hotel & 1 & 2009-05-15T14:29:15Z & 1 \\
\hline School & 1 & 2009-09-20T18:07:01Z & 2 & School & 1 & 2009-05-15T11:42:53Z & 2 \\
\hline Hospital & 2 & 2009-09-08T06:16:10Z & 1 & School & 1 & 2009-09-20T18:07:01Z & 2 \\
\hline Hotel & 2 & $2010-08-23 \mathrm{~T} 18: 25: 44 \mathrm{Z}$ & 3 & Hospital & 2 & 2009-09-08T06:16:10Z & 1 \\
\hline Hotel & 2 & 2009-08-23T18:25:13Z & 3 & Hotel & 2 & 2010-08-23T18:25:44Z & 3 \\
\hline Hotel & 2 & $2008-07-29 \mathrm{~T} 20: 50: 54 \mathrm{Z}$ & 3 & Hotel & 2 & 2009-08-23T18:25:13Z & 3 \\
\hline Farm & 3 & $2009-07-29 \mathrm{~T} 20: 50: 54 \mathrm{Z}$ & 11 & Farm & 3 & 2009-07-29T20:50:54Z & 1 \\
\hline
\end{tabular}

Figure 2. Visited locations log table

\subsection{Trust Calculation Module}

The interpersonal trust is the main factor that affects the influence diffusion in social networks. Forming trusted relationships in social networks is a new topic with lots of challenging questions. In the proposed model we employ the quantitative measurements introduce by Wagih et al. (2019). 
Weight: for each pair of friends $\left(f_{i}\right.$ and $\left.f_{j}\right)$ in the social network, the weight of their relation is calculated as the correlation between their attributes using Pearson Correlation Coefficient, and is given by,

$$
\text { Weight }(f i, f j)=P C C(\text { fiattr }, \text { fjattr })
$$

EdgeTrust: for each pair of friends $\left(f_{i}\right.$ and $f j$ ) in the social network, there is an interpersonal trust, and this is known as the edge trust value. Having a dataset of $m$ friends, we define the quantitative measure for edge trust as:

$$
\operatorname{EdgeTrust}(f i, f j)=\frac{W e i g h t(f i, f j)}{\sum_{x=1}^{m} \text { Weight }(f i, f x)}, \quad \mathrm{x}=1,2,3, \ldots . . \mathrm{j}, \ldots . ., \mathrm{m}
$$

Where Weight $\left(f_{i}\right.$ and $\left.f_{j}\right)$ denotes the weight of the relation between friends $\left(f_{i}\right.$ and $\left.f_{j}\right)$ and Weight $\left(f_{i}\right.$ and $f x$ ) denotes the weight of all the relations between $f_{i}$ and all directly connected friends in the network.

In social networks, some friends can have a degree of negative influence on other friends, especially if they do not trust. Since recommendation systems are based on trust among friends, thus we consider only the positive trust values among users. All relations with trust values less than an acceptable threshold are pruned creating a trusted web network.

$$
\operatorname{Imp}(\operatorname{EdgeTrust}(f i, f j))=\left\{\begin{array}{l}
1,<\text { Threshold } \\
0, \geq \text { Threshold }
\end{array}\right.
$$

Consider a social network with relations between four friends A, B, C and D. Figure 3 shows an example of weighted relation social network.

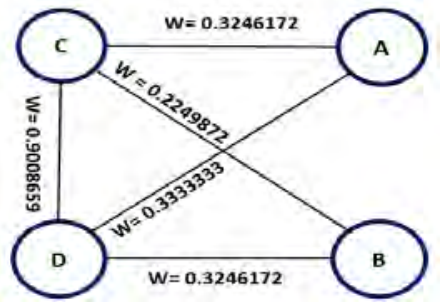

Figure 3. Weight values between friends in social network

Following the previous example, EdgeTrust between (Friend A, Friend C) is calculated as follows:

$$
\text { EdgeTrust }(f A, f C)=\frac{0.3246172}{0.3246172+0.3333333}
$$

NodeTrust: each friend in the social network has a trust value that he/she gains from other connected friends, this value is known as the node trust value. The higher the degree of trust value, the more trustworthy a friend is considered over the social network.

$$
\operatorname{NodeTrust}(f i)=\sum_{x=1}^{m} \operatorname{EdgeTrust}(f i, f j), \mathrm{x}=1,2,3, \ldots . \mathrm{j}, \ldots \ldots, \mathrm{m}
$$

Having a social network with completed EdgeTrust calculation, NodeTrust for (Friend A) is calculated as follows:

$$
\operatorname{NodeTrust}(f A)=0.565458095+0.414990168
$$

\subsection{Semantic-Based Influence Calculation Module}

In this module, we extend the trust calculation module by introducing new metrics, namely, Semantic Visited Places Finding (SVP), Semantic Edge Influence (SEdgeInf) and Semantic Node Influence (SNodeInf).

SemanticVisitedPlaces (SVP): in most datasets, locations are presented with their longitude and latitude values. In order to get the semantic translation for these spatial coordinates, a set of functions which are available in the geonames package in R programming language are used. Function GNfindNearby is used to retrieve a structured list of coordinates, places, countries and other information related to each check-in value. Figure 4 shows an example of semantic representation for a place with longitude equals to 35.24012 and latitude equals to 24.80927 . 
SVP $($ check_in
List $=$ GNfind, lat $)=$ List $\$$ geonames $\$$ flat, lng, radius $),$ radius $=0.01 \mathrm{~km}$

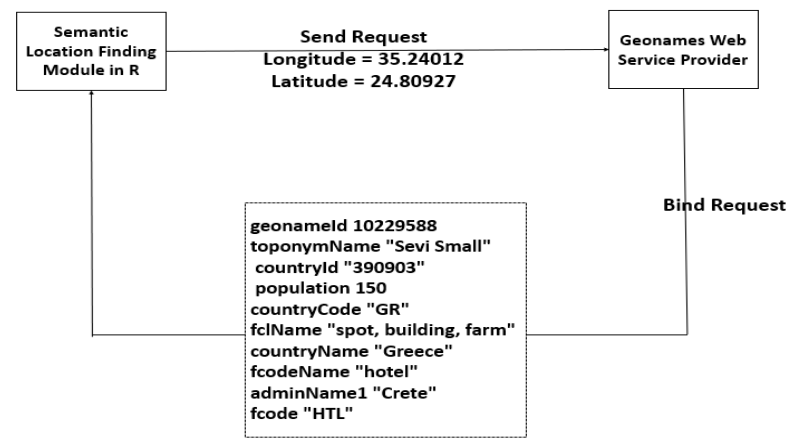

Figure 4. Semantic translation for geospatial coordinates

SemanticEdgeInfluence (SEdgeInf): each friend has a specific influence on each of his/her connected friends in the network which is known as the edge influence between every two friends. The influence $f_{i}$ has on $f_{j}$ is the product of the trust $f_{j}$ has on $f_{i}$ and the semantically based recommendation rate between $f_{i}$ and $f_{j}$. The semantic recommendation rate (SRecRate) is the degree to which two friends might share common interests through analyzing the semantics of the check-in data for each user.

$$
\begin{aligned}
& \operatorname{SEdgeInf}(f i, f j)=\operatorname{EdgeTrust}(f i, f j) * \operatorname{SRecRate}(f i, f j) \\
& \operatorname{SRecRate}(f i, f j)=\frac{\operatorname{sVP}(f i, f j)}{\operatorname{TSVP}(f i)} \\
& \operatorname{TSVP}(f i)=\{|\operatorname{SVP}(f i, \mathrm{X})|\}
\end{aligned}
$$

Following the previous example and having the SRecRate calculated between all friends, SEdgeInf between (Friend C, Friend A) is calculated as follows:

$$
\operatorname{SEdgeInf}(f C, f A)=0.493376325 * 0.00003198874
$$

SemanticNodeInfluence(SNodeInf): each friend in the social network has an influence degree on the whole network known as semantic node influence and is defined by the following equation.

$$
\text { SNodeInf }(f i)=\sum_{x=1}^{m} \operatorname{SEdgeInf}(f i, f x) * \operatorname{NodeTrust}(f i)
$$

\section{EVALUATION}

In this research, the experiments are conducted on two real social networks, namely, Brightkite and Weeplaces which are available for public use. The Experiments are performed to evaluate the efficiency and accuracy of STRUM model against other conventional models. The Experiments highlighted the significance of adding semantic information in location recommendation services as well as in discovering the influential nodes in the network. Multiple layers of semantics are added to the dataset and after each layer, friends' influence is calculated and a list with the top influential friends is created for each friend in the network. Layers of semantics are added till we reach the highest abstract meaning for locations as shown in Figure 5. 


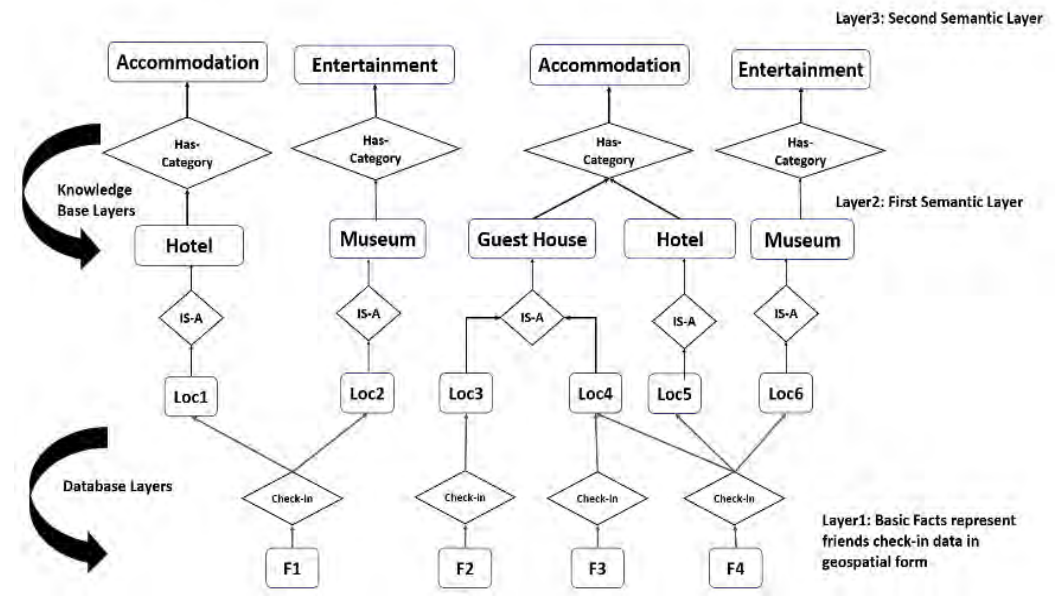

Figure 5. Semantic summary graph

The STRUM model is compared to the SIMBA algorithm proposed by Wagih et al. (2019), the influence-based algorithm proposed by Bagci et al. (2016) and weight-based algorithm proposed by Hangal et al. (2010) as shown in Figure 6. Remarkable enhancements are observed with each added layer of semantics. The proposed algorithm outperforms SIMBA by $15 \%$, algorithm Bagci et al. (2016) by $30 \%$ and algorithm Hangal et al. (2010) by $40 \%$ in recommendation accuracy.

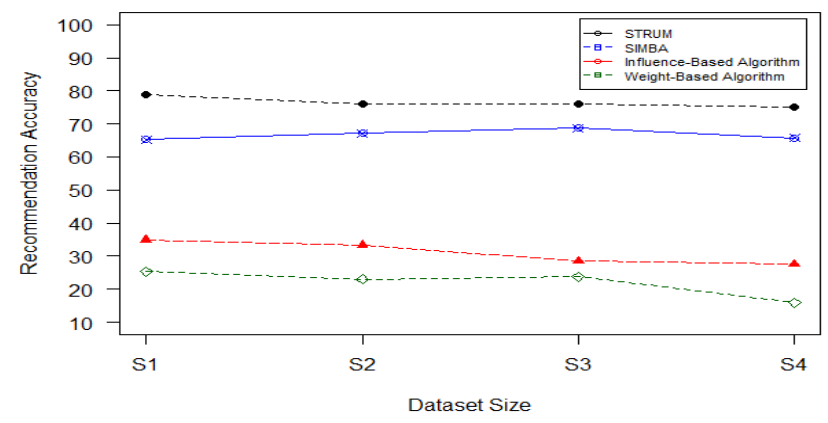

Figure 6. A recommendation accuracy comparison between STRUM, SIMBA, influence-based algorithm and weight-based algorithm.

We also compare the efficiency in detecting the influential nodes between STRUM, and other conventional algorithms as the degree centrality measure, betweenness centrality measure, closeness centrality measure, K-Core decomposition and CLC algorithm and the results are shown in Figure 7. 

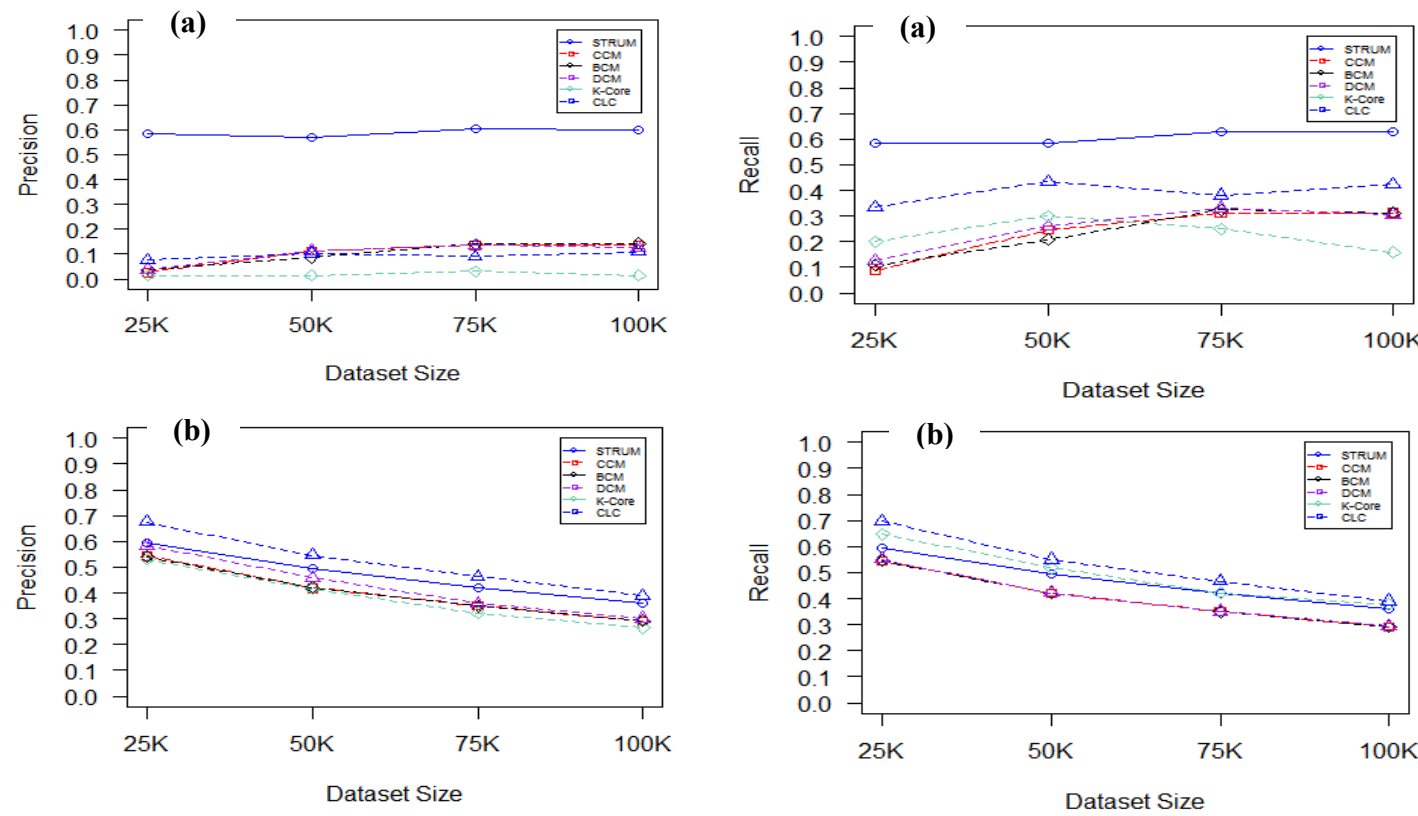

Figure 7. A Performance comparison between STRUM, DCM, BCM, CCM, K-core decomposition and CLC in terms of precision and recall for (a) Brightkite and (b) Weeplaces datasets

The experiments show that nodes with highest influence in the network might not have the highest power in recommendation (e.g. in STRUM, almost 35\% of the recommendation comes from friends who have direct influence on their friends rather than having a significant influence on the network as shown in Figure 8.

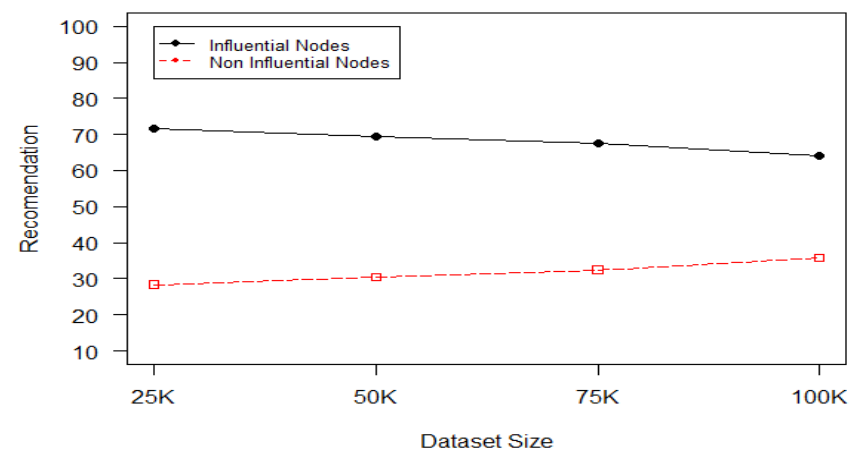

Figure 8. Recommendation percentage from influential and non-influential nodes

\section{CONCLUSIONS AND FUTURE WORK}

In this paper, we introduce a model for detecting influential nodes and enhancing the location recommendation process. The proposed model utilizes the trust concept between participants of social networks. It considers both the social and spatial information related to each friend in the network. We use two real datasets from different LBSNs for conducting our experiments; namely, Brightkite and Weeplaces. We proved experimentally that the proposed model outperforms relevant prior works for all datasets. Several future enhancements are to be considered related to the presented work. The proposed model can be extended to include more semantic information as well as indirect connections in social networks (friend of friend). 


\section{REFERENCES}

Alvarez-Hamelin, J. I., Dall'Asta, L., Barrat, A. and Vespignani, A. (2005). Large scale networks fingerprinting and visualization using the $k$-core decomposition. In Proceedings of the 18th International Conference on Neural Information Processing Systems, NIPS'05, pp. 41-50, Cambridge, MA, USA, MIT Press.

Ben Amor, S., Ben Romdhane, L. and Harzallah, M. (2016). Nouvelle approche sémantique pour la détection des communautés dans un réseau social. In IC2016: Ingenierie des Connaissances, Montpellier, France.

Bonchi, F. (2011). Influence propagation in social networks: A data mining perspective. In 2011 IEEE/WIC/ACM International Conferences on Web Intelligence and Intelligent Agent Technology, volume 1, pages 2-2.

Davoudi, A. and Chatterjee, M. (2018). Social trust model for rating prediction in recommender systems: Effects of similarity, centrality, and social ties. Online Social Networks and Media, pp.1-11.

Hafiene, N. and Karoui, W. (2017). A new structural and semantic approach for identifying influential nodes in social networks. 2017 IEEE/ACS 14th International Conference on Computer Systems and Applications (AICCSA), pp, 1338-1345.

Bagci, H. and Karagoz, P. (2011). Context-Aware Friend Recommendation for Location Based Social Networks Using RandomWalk, Proceedings of the 25th International Conference Companion on World Wide Web, pp. 531-536,

978-1-4503-4144-8.

Hangal, S., MacLean, D., Lam, M. S. and Heer, J. (2010). All friends are not equal: Using weights in social graphs to improve search. In Workshop on Social Network Mining \& Analysis, ACM KDD.

Jaouadi, M. and Ben Romdhane, L. (2016). Din: An efficient algorithm for detecting influential nodes in social graphs using network structure and attributes. In 2016 IEEE/ACS 13th International Conference of Computer Systems and Applications (AICCSA), pp. 1-8.

Kim, H., Beznosov, K. and Yoneki, E. (2014). Finding influential neighbors to maximize information diffusion in twitter. In Proceedings of the 23rd International Conference on World Wide Web, WWW'14 Companion, pp. 701-706, New York, NY, USA.

Landherr, A., Friedl, B. and Heidemann,J. (2010). A critical review of centrality measures in social networks. Business \& Information Systems Engineering, pp. 371-385.

Martınez, D. C., Janowicz, K., and Hitzler, P. (2012). A logical geo-ontology design pattern for quantifying over types. in SIGSPATIAL 2012 International Conference on Advances in Geographic Information Systems (formerly known as GIS), SIGSPATIAL'12, Redondo Beach, CA, USA, pp. 239-248.

Rodríguez Hernández, M. C., Ilarri, S., Trillo, R., and Hermoso, R. (2015). Location-aware recommendation systems: Where we are and where we recommend to go. In CEUR Workshop Proceedings, pp. 1-8.

Song, H., Pei, Q., Xiao, Y., Li, Z. and Wang, Y. (2017). A novel recommendation model based on trust relations and item ratings in social networks. In 2017 International Conference on Networking and Network Applications (NaNA), pp. 17-23.

Wagih, H.M., Mokhtar, H.M.O. and Ghoniemy, S.S. (2019). SIMBA: A Semantic-Influence Measurement Based Algorithm for Detecting Influential Diffusion in Social Networks. The 13th IEEE International Conference on Semantic Computing, pp. 178-182.

Wei, H., Pan, Z., Hu, G., Zhang, L., Yang, H., Li, X. and Zhou, X. (2018). Identifying influential nodes based on network representation learning in complex networks. In PloS one, Public Library of Science.

Wang, J., Jiang, W., Li, K., and Li, K. (2017). Detecting influential nodes incrementally and evolutionarily in online social networks. In 2017 IEEE International Symposium on Parallel and Distributed Processing with Applications and 2017 IEEE International Conference on Ubiquitous Computing and Communications (ISPA/IUCC), pp. 182-189.

Yang, B., Lei, Y., Liu, J. and Li, W. (2017). Social collaborative filtering by trust. IEEE Transactions on Pattern Analysis and Machine Intelligence, 39(8), pp. 1633-1647.

Zhao. X., Liu. F., Wang, J., and Li, T. (2017). Evaluating influential nodes in social networks by local centrality with a coefficient. ISPRS International Journal of Geo-Information. 